\title{
Quararibea calycoptera (Malvaceae), una nueva especie de los bosques muy húmedos del Pacífico de Ecuador y Colombia
}

\section{Acta Botanica Mexicana}

\author{
Quararibea calycoptera (Malvaceae), a new species from \\ the Pacific wet forests of Ecuador and Colombia
}

\author{
José Luis Fernández-Alonso1,3 (iD), Xavier Cornejo²
}

\begin{abstract}
Resumen:
Antecedentes y Objetivos: El género de árboles Quararibea (Malvaceae), propio del Nuevo Mundo, agrupa a unas 60 especies con distribución centrada en el norte de Sudamérica y sur de Mesoamérica. Como parte del trabajo de revisión que se está llevando a cabo en un grupo morfológico de Quararibea que presenta columna estaminal digitada, en este trabajo se describe una nueva especie proveniente de los bosques muy húmedos del Pacífico de Ecuador y Colombia y se propone su categorización IUCN.

Métodos: Se estudió un grupo de colecciones de herbario de Quararibea, provenientes de los bosques muy húmedos del norte de Ecuador, que presentaban algunas divergencias morfológicas con respecto a las especies descritas en este género. Se realizó la diagnosis morfológica y se delimitó la distribución geográfica de estas plantas, proponiéndose aquí como un taxón nuevo. Asimismo, se hace una propuesta preliminar de categorización, de acuerdo con los criterios de la Lista Roja de la UICN.

Resultados clave: Se describe y documenta gráficamente la nueva especie Quararibea calycoptera, como especie afín a Q. grandifolia y a Q. cornejoi, con las que se compara. La nueva especie se separa morfológicamente de ambas por los siguientes caracteres: la consistencia de la lámina, la longitud del pedicelo floral y bractéolas y la forma y tamaño del cáliz floral.

Conclusiones: Dentro de lo que se había venido identificando como Quararibea grandifolia en el Occidente de Ecuador, con base en el estudio de nuevas colecciones de los bosques muy húmedos de Esmeraldas, pudo diagnosticarse lo que representa una nueva especie asignable al pequeño grupo de especies de Quararibea, con columna estaminal digitada y cálices alados, en proceso de revisión.
\end{abstract}

Palabras clave: Esmeraldas, Matisia, Nariño, Neotrópico, Quararibea grandifolia, taxonomía.

\section{Abstract:}

Background and Aims: The Quararibea (Malvaceae) tree genus, typical of the New World, groups about 60 species with a distribution centered in northern South America and southern Mesoamerica. As part of the revision work that is currently being carried out in a morphological group of Quararibea that presents a digitate staminal column, a new species from the wet forests of the Pacific of Ecuador and Colombia is described here, and its IUCN categorization is proposed.

Methods: A group of herbarium collections of Quararibea from the wet forests of northern Ecuador, that showed some divergences with respect to the described species, was studied. The morphological diagnosis was realized, and the geographical distribution of these plants was delimited, which are described here as a different new taxon. Based on the known localities for the new species and in relation to its conservation, a first categorization proposal is made in accordance with the criteria of the IUCN.

Key results: The new species Quararibea calycoptera, related to $Q$. grandifolia and $Q$. cornejoi, is described and graphically documented. It is separated morphologically from both species by the following characters: the consistency of the blade, the length of the floral pedicel and bracteoles, and the shape and size of the floral calyx.

Conclusions: Within what had been identified as Quararibea grandifolia in western Ecuador, based on the study of new collections from the wet forests of Esmeraldas, it was possible to diagnose what represents a new species assignable to the small group of Quararibea species that has a digitate staminal column and winged calyces, currently under revision.

Key words: Esmeraldas, Matisia, Nariño, Neotropics, Quararibea grandifolia, taxonomy.

${ }^{1}$ Real Jardín Botánico CSIC, Departamento de Biodiversidad y Conservación, Claudio Moyano 1, 28014 Madrid, España.

2Universidad de Guayaquil, Facultad de Ciencias Naturales, Departamento de Botánica, Herbario GUAY, Av. Raúl Gómez Lince s.n. y Av. Juan Tanca Marengo (campus Mapasingue), 09-01-10634 Guayaquil, Ecuador.

${ }^{3}$ Autor para la correspondencia: jlfernandeza@rjb. csic.es
Recibido: 24 de agosto de 2021.

Revisado: 4 de octubre de 2021.

Aceptado por Marie-Stéphanie Samain: 27 de octubre de 2021.

Publicado Primero en línea: 19 de noviembre de 2021. Publicado: Acta Botanica Mexicana 128 (2021).

cc) (7) (8) Este es un artículo de acceso abierto cc. Atribución-No Comercial (CC BY-NC 4.0 Internacional).
Citar como: Fernández-Alonso, J. L. y X. Cornejo. 2021. Quararibea calycoptera (Malvaceae), una nueva especie de los bosques muy húmedos del Pacífico de Ecuador y Colombia. Acta Botanica Mexicana 128: e1960. DOI: https://doi.org/10.21829/ abm128.2021.1960

e-ISSN: $2448-7589$ 


\section{Introducción}

El género de árboles Quararibea Aubl. s.s. (Malvaceae), propio del Nuevo Mundo, se distribuye ampliamente en los bosques de la franja intertropical desde México y las Antillas hasta Bolivia y Argentina (Alverson, 1989; Fernández-Alonso, 1999). Dentro de este rango geográfico existen zonas con mayor diversificación morfológica asociada a una mayor diversidad de especies: una en el norte de Sudamérica (Colombia y Perú, ca. 35 especies), donde aún falta mucho trabajo de campo por hacer, y otra en el sur de Mesoamérica (Costa Rica y Panamá, 18 especies), donde la exploración y el estudio de este grupo se encuentra más adelantado (Cascante-Marín et al., 2018; Fernández-Alonso, 2021a). Incluyendo las 20 especies de Quararibea descritas en este siglo, la diversidad del género se cifra actualmente en unas 60 , algunas de ellas pobremente documentadas hasta ahora (Cascante-Marín et al., 2018; Fernández-Alonso, 2021a, b). Para Colombia se han indicado en la literatura 22 especies (Fernández-Alonso, 2021a, b) a las que hay que añadir la que se describe en este trabajo y algunas más que están en proceso de descripción (Fernández-Alonso, en prep.). En lo que respecta a Ecuador, el número actual de especies conocidas asciende a diez, incluyendo la recientemente descrita Q. cornejoi Fern.Alonso (Fernández-Alonso, 2021b) y la que ahora se propone.

Como continuación del trabajo de revisión que actualmente se ultima en el grupo de 12 especies de Quararibea que presentan columna estaminal claramente digitada (Fernández-Alonso, en prep.), se propone aquí una nueva especie asignable al subgrupo que presenta cálices alados (cinco especies), que se encuentra diversificado en los bosques del Pacífico de Ecuador y Colombia. Para dar una idea del escaso conocimiento de este grupo de especies hasta fechas muy recientes, la especie más conocida Quararibea grandifolia (Little) Cuatrec. fue tratada inicialmente como especie del género Matisia Bonpl. y luego asignada con reservas, a la sección Calyculatae Fern. Alonso del género Matisia (Little, 1948; Fernández-Alonso, 2001a; 2016). La presencia de cinco lóculos en el ovario, referida en el protólogo de Matisia grandifolia Little (Little, 1948), que correspondería a un ovario pentacarpelar, no ha podido ser confirmada de acuerdo con nuestras observaciones en diferente material preservado en líquido. Por otra parte, el que no cuente el protólogo con información sobre el número de pirenos en el fruto (faltaban en las muestras originales estudiadas por Little), genera aún más dudas sobre la presencia real de cinco carpelos en el ovario de esta especie, que nunca fueron detectados en colecciones posteriores revisadas, en todo este "grupo grandifolia" del género Quararibea. El análisis de las nuevas recolecciones de campo, obtenidas en este grupo de plantas, permitió arrojar luz sobre variabilidad en algunos caracteres del androceo y gineceo y proponer un nuevo taxón relacionado.

\section{Materiales y Métodos}

Se estudiaron colecciones del género Quararibea depositadas principalmente en herbarios de Colombia, Ecuador y los Estados Unidos de América: COL, GH, GUAY, MO, NY, PSO, QCNE y US (acrónimos de acuerdo con Thiers, 2020). Se consultaron imágenes complementarias de material de herbario y tipos nomenclaturales en la base de datos Tropicos del Missouri Botanical Garden (TROPICOS, 2021) y en la base de datos Global Plants de JSTOR (2021). Estas colecciones fueron la base del estudio morfológico y de la distribución geográfica de la nueva especie descrita, así como de otras especies relacionadas que se comentan en este trabajo. En este estudio morfológico se siguió la metodología clásica ya indicada en contribuciones anteriores (Fernández-Alonso, 1996; 2001a) y en la terminología descriptiva general se siguió a Font Quer (1985) y a Harris y Harris (2001). Como parte de este estudio, se tomaron fotografías de acercamiento (tricomas, cortes transversales y detalles de algunas estructuras florales) con una cámara Canon PowerShot SX260HS (Amstelveen, Holanda) y también con una lupa binocular Nikon SMZ645 (Japón), acoplada, con lente de 100x.

\section{Resultados}

\section{Taxonomía}

Quararibea calycoptera Fern.Alonso \& Cornejo, sp. nov., Figs. 1, 2, 3, 4.

TIPO: ECUADOR. Provincia Esmeraldas, Reserva Mache-Chindul, Cuchilla de Bunca, $470 \mathrm{~m}$, bosque muy húmedo tropical intervenido, 29.IV.2003, árbol 10 m, fl, C. Boni- 
faz y X. Cornejo 5019 (holotipo: GUAY 12052!, isotipos: COL 492634 !, 508075!).

Quararibea calycoptera resembles Quararibea grandifolia, but can be well recognized because it presents membranous blades (vs. chartaceous blades); longer floral pedicel (8-18 $\mathrm{mm}$ vs. 4-7 $\mathrm{mm}$ ), with 3 erect-patent, falcate bracteoles, 5-11 $\mathrm{mm}$ long, arranged along the pedicel (vs. appressed bracteoles, broadly subulate, 3-6 mm long, distally grouped at the base of the calyx); flower bud narrowly spindle-shaped and long apiculate (vs. ovoid-elliptic-shaped, shortly apiculate); calyx broadly tubular-campanulate, 27-31 × 10-12 mm distally (vs. narrowly tubular-campanulate, 20-25 $\times 7-9 \mathrm{~mm}$ ), wings on the flower bud and the calyx broad 2-3 $\mathrm{mm}$ tall (vs. wings 1-1.5 $\mathrm{mm}$ tall).

Árboles pequeños, hasta 5-10 m, tronco recto; corteza lisa, blanco-verdosa, leño color crema; ramas de primer orden delgadas (generalmente de menos de $8 \mathrm{~cm}$ de diámetro), verticiladas; ramas terminales glabras, articuladas, aspecto ligeramente carnoso; entrenudos cortos, 1-4(-5) $\mathrm{cm}$, a menudo con orificios circulares -de hormigas-,y segmentos huecos en su interior (domacios); corteza verde o amarillenta, frecuentemente con lenticelas muy conspicuas; yemas apicales generalmente con un fascículo de primordios foliares estipulares, subulado-falcados, 5-10 × 1-2 $\mathrm{mm}$, densamente cubiertos de indumento color marrón, lepidoto muy fino; hojas alternas, dísticas; peciolo 4-10(12) $\mathrm{mm}$ de longitud, 3-4 $\mathrm{mm}$ de diámetro; estípulas triangular-subuladas, 3-5 $\times$ 2-3 $\mathrm{mm}$ en su base, prontamente caducas, dejando cicatrices linear-oblicuas conspicuas en las ramas; láminas membranáceas, anchamente elípticas u oblanceoladas, $35-55 \times 18-28 \mathrm{~cm}$, limbo generalmente inequilátero, estrechado gradualmente hacia la base y redondeado en la zona de unión al peciolo; apiculado, con apículo estrechamente triangular, $1.5-2 \mathrm{~cm}$; margen entero u ondulado, más raramente sinuoso-dentado; haz con venas impresas y aspecto levemente reticulado; envés con vena media muy resaltada, 9-13 venas secundarias a cada lado; en la cara abaxial muy resaltadas; venas de tercer y cuarto orden reticuladas; haz glabro, envés con indumento disperso estrellado-pubérulo; flores en ramas jóvenes, solitarias y opuestas a las hojas, raramente hasta 2-3 en ra-

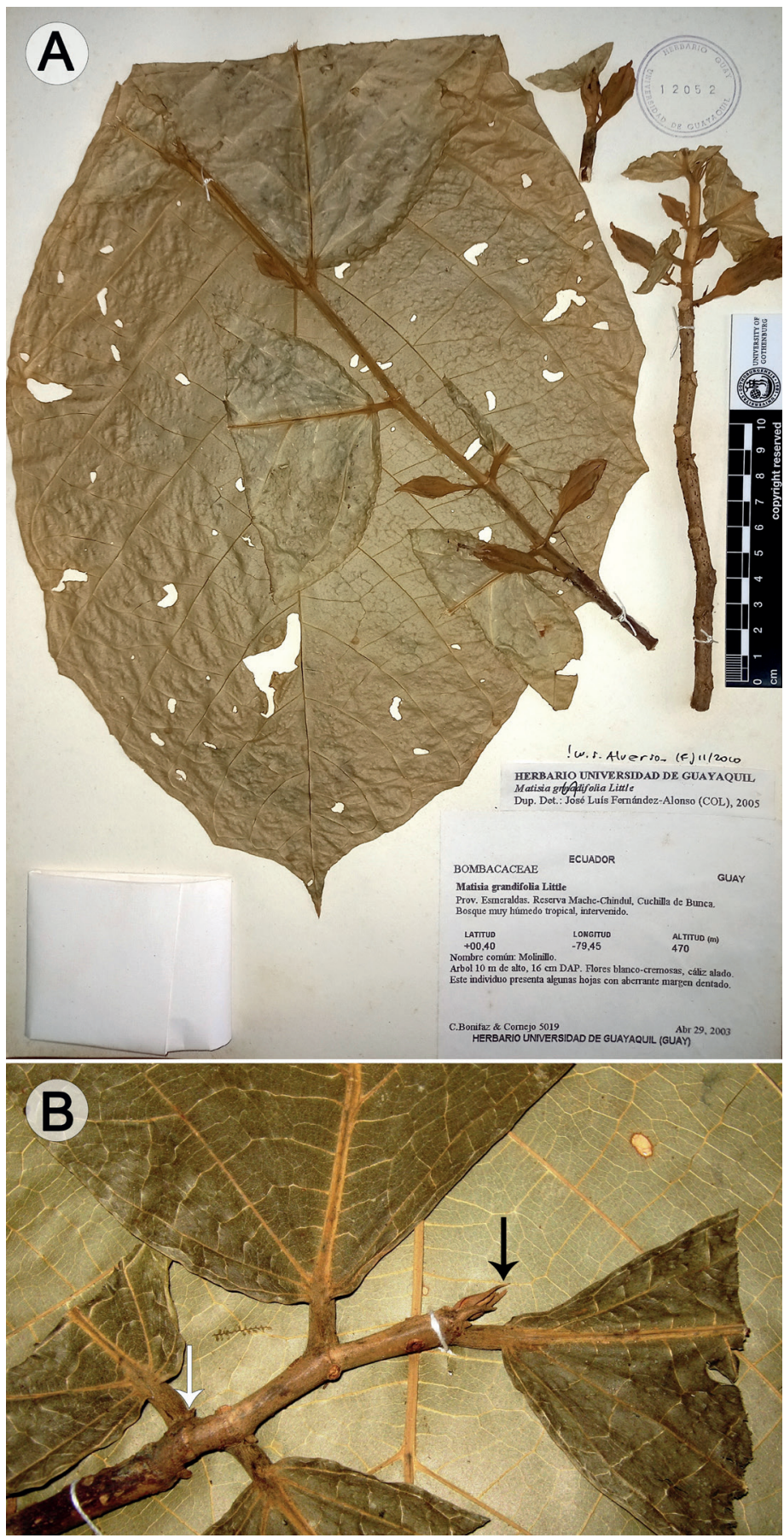

Figura 1: Quararibea calycoptera Fern.Alonso \& Cornejo. A. sección de una rama terminal (C. Bonifaz y X. Cornejo 5019, GUAY12052); B. detalle de la rama terminal con las cicatrices estipulares (flecha blanca), la yema terminal con catáfilos (flecha negra) y la base inequilátera de las láminas (X. Cornejo y C. Bonifaz 6469, COL 458361).

mitas muy cortas opuestas a las hojas; flor (35-)37-45 mm de longitud; pedicelo (8-)9-18 × 2-3 mm, longitudinalmente alado-anguloso en su mitad distal (prolongación de las alas calicinas), con fino indumento marrón, lepidoto o estrella- 

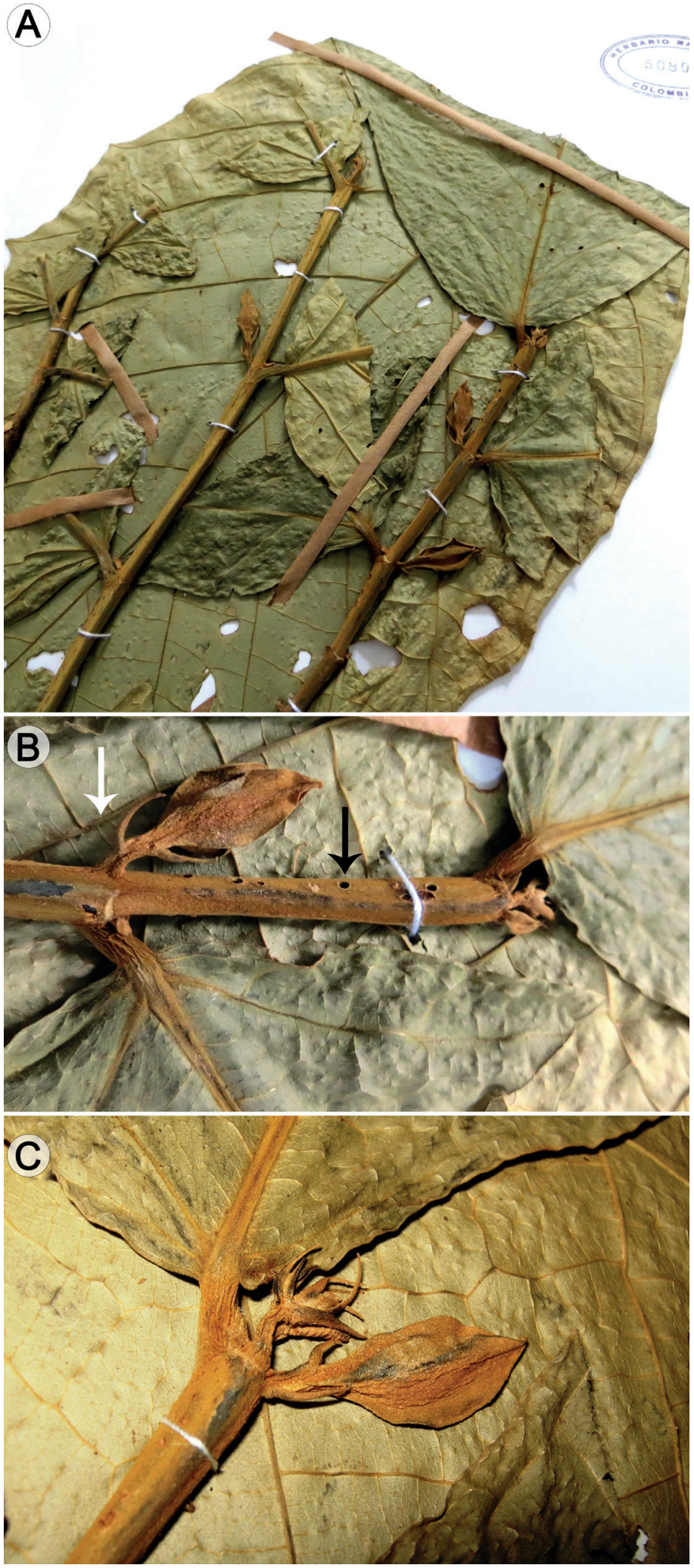

Figura 2: Quararibea calycoptera Fern.Alonso \& Cornejo. A y B. detalle de los pedicelos florales con bractéolas distantes y falcadas (flecha blanca) y de las perforaciones de los entrenudos huecos (flecha negra), debidas a hormigas asociadas (C. Bonifaz y X. Cornejo 5019, COL 508075); C. detalle de un botón floral opuesto a la hoja y el eje terminal de crecimiento con estípulas jóvenes. (C. Bonifaz y X. Cornejo 5019, COL 409634). do-lepidoto; con 3 bractéolas persistentes, dispuestas de modo disperso a lo largo del pedicelo, bractéolas erectopatentes, incurvo-falcadas, agudas, 5-11 mm de longitud, 2-3 $\mathrm{mm}$ de ancho en su zona basal; glabrescentes o con fino indumento lepidoto; botón floral de estrechamente romboide a fusiforme-apiculado, 25-35 $\mathrm{mm}$ de largo y 8-10 $\mathrm{mm}$ de ancho en su zona media, alas longitudinales muy marcadas, 2-3 $\mathrm{mm}$ de alto, gradualmente adelgazadas en la zona de confluencia del pedicelo y prolongadas también en la zona apical, indumento finamente lepidoto; cáliz de verdoso pálido a café, anchamente tubular-campanulado, 27-31 × 10-12 mm en la zona distal, tubo $19-24 \mathrm{~mm}$ de longitud, 2-4 lobado-rasgado irregularmente en el tercio o cuarto distal; lóbulos 2-7 mm de longitud, 5 alas longitudinales, agudas, muy notorias, 2-3 mm de alto, raramente alternando con otras 3-4 alas incipientes, en su cara externa densamente cubierto de indumento muy fino estrellado, en su cara interna con indumento seríceo acostado, densamente dispuesto; corola, 5 pétalos, color blanco o crema, tornándose ligeramente rojizos o asalmonados al final de la antesis, erecto-patentes o patentes, $35-42 \mathrm{~mm}$ de largo, 4-6 $\mathrm{mm}$ de ancho en la zona distal, 2-3 $\mathrm{mm}$ de ancho en el tercio basal, estrechamente oblanceolados, unguiculados en la base y obtusos distalmente, glabros en su cara externa, indumento estrellado-tomentoso muy corto en la zona distal; androceo con columna estaminal blanca (en fresco), cilíndrica, estriada longitudinalmente, $35-40 \mathrm{~mm}$ de longitud, 2-2.5 mm de grosor, finamente pubescente en su zona apical, con 5 ramas estaminales, 6-10 $\mathrm{mm}$ de longitud, 6-7 tecas en cada rama; ovario subesférico, ca. $4 \mathrm{~mm}$ de longitud, generalmente 4-locular, 2-4 carpelos desarrollados, 2 óvulos por carpelo y evidencia de 1-2 carpelos abortados; estilo recto, $35-41 \mathrm{~mm}$ de longitud, indumento estrellado tomentoso muy fino; estigma amarillento, ensanchado, 2-3 $\mathrm{mm}$ de diámetro, 5-lobulado, lóbulos obtusos; pedúnculo fructífero conservando las bractéolas, indumento denso marrón, finamente estrellado; frutos con el cáliz persistente y ligeramente acrescente, $33-36 \times 30 \mathrm{~mm}$, recubriendo en gran parte al fruto e irregularmente rasgado en su tercio distal, con el mismo indumento marrón del pedicelo, fruto propiamente dicho, amarillento o amarillo, ovoide, 35-38 × 30-34 mm, (2-)3-4 pirenos leñosos desarrollados. 


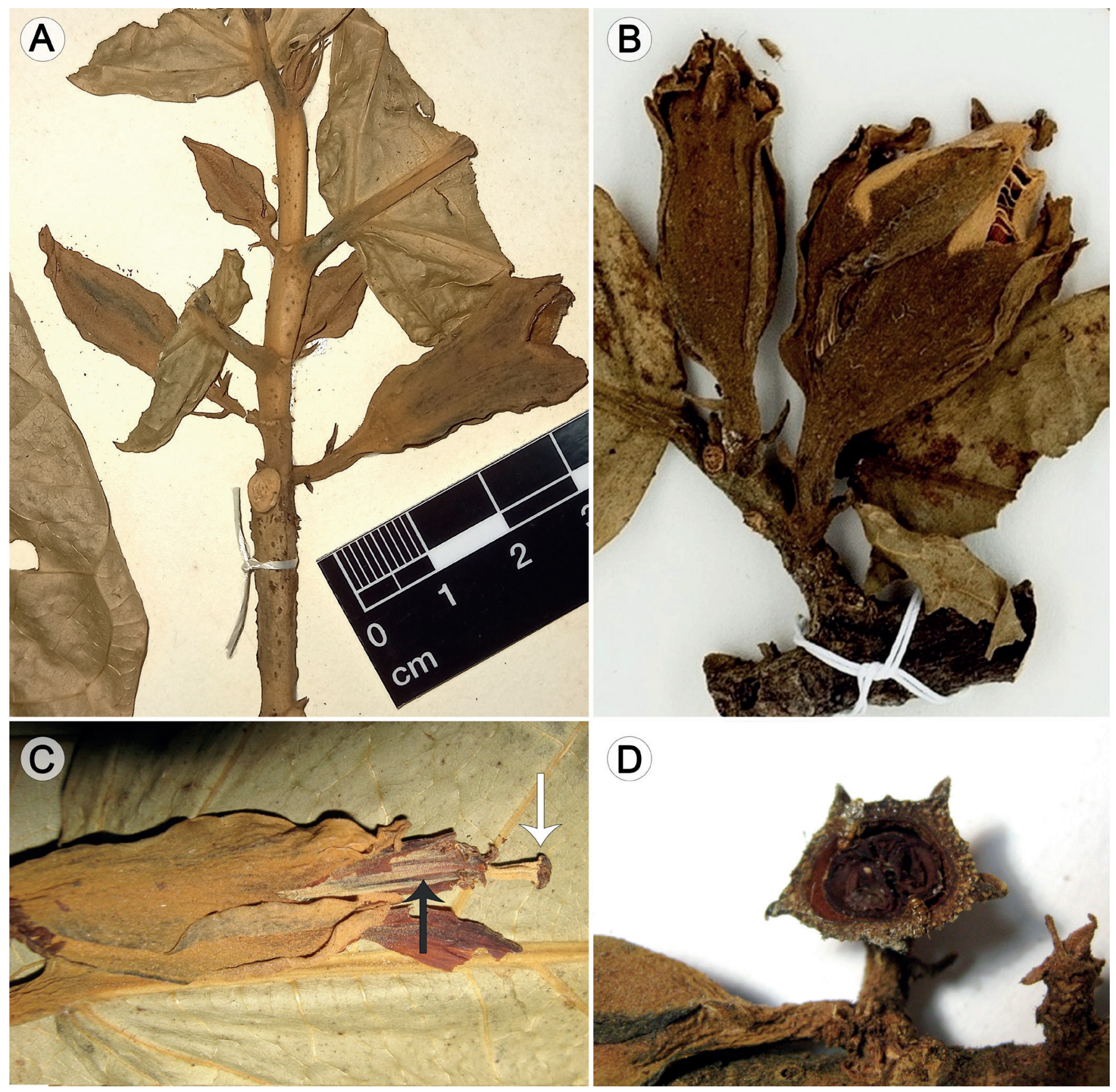

Figura 3: Quararibea calycoptera Fern.Alonso \& Cornejo. A. rama terminal mostrando botones florales y un cáliz maduro anchamente tubularcampanulado; B. detalle de un fruto parcialmente recubierto por el cáliz persistente alado y rasgado; $\mathrm{C}$. detalle de una flor vista lateralmente con la porción distal de la columna estaminal (flecha negra) y del estilo-estigma (flecha blanca) que la sobrepasa ligeramente; D. corte transversal de un botón floral (visto en aumento con lupa binocular) mostrando las cinco alas principales en el cáliz, dos carpelos fértiles y restos de otros dos, no desarrollados en el ovario. Fotografías: A y C, C. Bonifaz y X. Cornejo 5019 (A, del holotipo GUAY 12052; C, del isotipo COL 492634); B, J. L. Clark y C. Watt 819 (NY 2609208); D, W. Palacios 13642 (COL 453664).

Distribución y ecología: esta especie, hasta ahora, solo se conoce de los bosques muy húmedos tropicales (BmhT) y de su zona de transición con los bosques muy húmedos premontanos (BmhPM) de Esmeraldas, en el sector norte del Pacífico de Ecuador y zonas limítrofes de
Colombia, cuenca del Río Mira, departamento Nariño (Fig. 5). Se encuentra principalmente en bosques primarios en las proximidades de ríos y en relieve de colinas disectadas, desde el nivel del mar hasta 600-650 m. Su presencia en Colombia se basa en un único registro del corregimiento de 


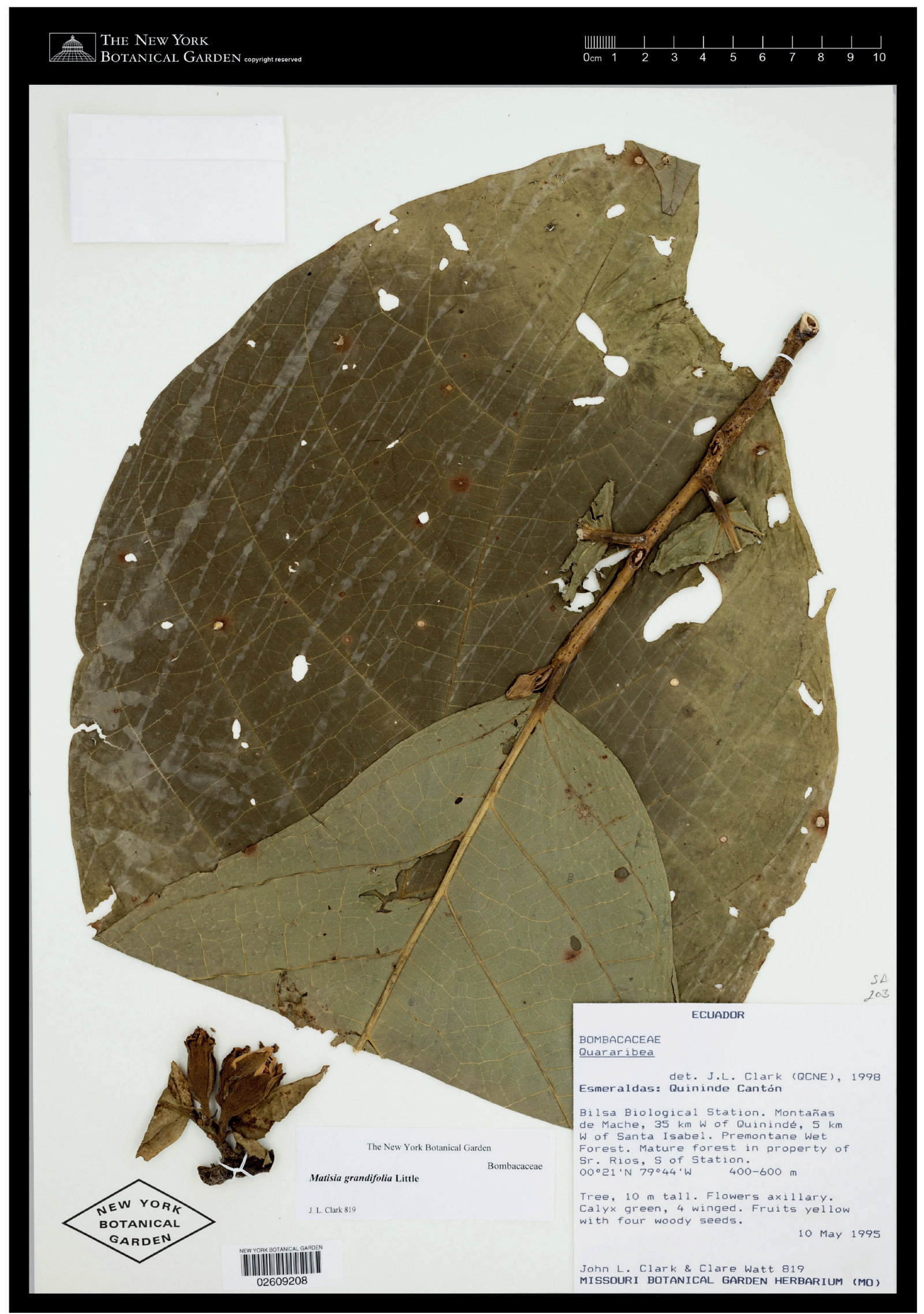

Figura 4: Quararibea calycoptera Fern.Alonso \& Cornejo. Espécimen J. L. Clark y C. Watt 819 (NY 2609208), árbol de 10 m, con frutos, proveniente de la Estación Biológica Bilsa, Quinindé, Esmeraldas, Ecuador. 
Espriella, Tumaco, muy próximo a la frontera ecuatoriana (Fig. 5), con bosques similares a los de Esmeraldas. Toda esta región comparte varias especies de Malvaceae, algunas de distribución restringida: Matisia alata Little, M. longipes Little, M. giacomettoi Romero, M. soegengii Cuatrec., Phragmotheca siderosa Cuatrec. y Quararibea casasecae Fern.Alonso \& Castrov. (Fernández-Alonso, 2001a; 2021a; Fernández-Alonso y Castroviejo, 2001).

Fenología: florece en febrero-abril y julio-septiembre; fructifica en mayo. Aparenta tener producción continuada de flores a lo largo del año, asociada quizás al permanente clima muy húmedo sin periodos de escasez de lluvias.

Nombre común: "molinillo" en la región de MacheChindul en Ecuador, es una denominación usual en el norte de Sudamérica, al igual que sus variantes ortográficas "molenillo" y "bolenillo" para otras especies de este género como $Q$. tulekunae Fern.Alonso y Q. cornejoi (FernándezAlonso, 2001b; 2021b).

Etimología: el nombre de la especie hace referencia al cáliz notoriamente alado. Esta condición se presenta también en otras especies del género Quararibea, como Q. pterocalyx Hemsl., Q. cogolloi Fern.Alonso y Q. rangeli Fern.Alonso, entre las especies con columna estaminal no digitada; además de $Q$. grandifolia y $Q$. cornejoi, especies con columna estaminal digitada con las que se compara.

Estatus de conservación (IUCN): esta especie se conoce solo de cinco localidades en el departamento Esmeraldas, norte de Ecuador, y de una localidad limítrofe en territorio colombiano (Fig. 5), que son regiones donde ha habido una importante alteración antrópica del hábitat de bosque original en las últimas cinco décadas (Little y Dixon, 1969; Dodson y Gentry, 1991; Jørgensen y León, 1999; Cornejo, 2011). Dado que su área de extensión de presencia es de ca. $4500 \mathrm{~km}^{2}$ y su área de ocupación real probablemente inferior a $2000 \mathrm{~km}^{2}$, se propone para esta especie la categoría de: En Peligro, EN-B2 ab(iii) (IUCN, 2019).

Especímenes adicionales examinados: COLOMBIA. Departamento Nariño, municipio Tumaco, corregimiento de Espriella, granja Las Delicias, bosque cerca del río Caunapí, $150 \mathrm{~m}, 15 . V .1994$, arbolito $2.5 \mathrm{~m}$, botones, B. R. Ramírez 6346 (PSO 30477). ECUADOR. Provincia Esmeraldas, municipio Quinindé, Estación Biológica Bilsa, montañas de Mache, $35 \mathrm{~km}$ al W de Quinindé, $5 \mathrm{~km}$ al W de Santa Isabel, $00^{\circ} 21^{\prime} \mathrm{N}, 79^{\circ} 44^{\prime} \mathrm{W}, 400-600 \mathrm{~m}, \mathrm{BmhPM}$ maduro, 10.V.1995, árbol 10 m, fr, J. L.Clark y C. Watt 819 (COL 453668, COL 453678, COL 543705, MO 5339440, NY 26009208, US 3404404). Municipio Bravito, entre Estero Mongón y Estero Sabaleta, $00^{\circ} 35^{\prime} \mathrm{N}, 79^{\circ} 02^{\prime} \mathrm{W}, 600 \mathrm{~m}$, BmhT primario, 10.IX.1998, árbol 3 m, fl, X. Cornejo y C. Bonifaz 6469 (COL 458361, GUAY 10214); Río Pambil, Estero Capulí, 25 m, BmhT de galería, 7.VI.1966, árbol $10 \mathrm{~m}, 27 \mathrm{~cm}$ dap, fl, $C$. Epling y C. Jativa 1084 (US 2534020, fl, US 2707038); loc. cit., Río Pambil, Estero Capulí, 20 m, BmhT, 7.VI.1966, árbol 10 m, fl, C. D. Játiva 304 (MO 3407200, US 2644855); Quinindé, carretera a Herrera - Los Mangos, cabecera del río Aguacatal, $00^{\circ} 19^{\prime} \mathrm{N}, 79^{\circ} 46^{\prime} \mathrm{W}, 550 \mathrm{~m}$, bosque primario sobre colinas disectadas, 24.II.1995, árbol $5 \mathrm{~m}$, fl, W. Palacios 13624 (COL 453664, MO 5179101, QCNE).

Notas taxonómicas: Se constata la presencia de entrenudos huecos en la parte distal de las ramas jóvenes terminales, que muestran orificios circulares de hormigas (domacios), ya referida en la descripción de $Q$. calycoptera, con base en lo observado en parte de las muestras estudiadas (así en Bonifaz y Cornejo 5019). Este tipo de interacción planta-insecto, en la que las hormigas defienden activamente a la planta, fue observada con características muy similares en otra malvácea arborescente, Ceiba pentandra (L.) Gaertn., por el primer autor de este trabajo, en Veraguas, en el litoral Pacífico de Panamá (Ibáñez et al., 2005).

\section{Discusión}

Este trabajo es un avance puntual de una revisión próxima a publicarse, de un grupo de 12 especies del género Quararibea, que presenta androceo con columna estaminal digitada (Fernández-Alonso, en prep.). Por este motivo, solo se hace referencia escueta aquí a sus afinidades más cercanas (con dos especies) sin incluir una clave de identificación para todo el grupo. De la especie considerada más afín, $Q$. grandifolia, a la que tradicionalmente se había asimilado en las colecciones de herbario (Fernández-Alonso, 2001a), 


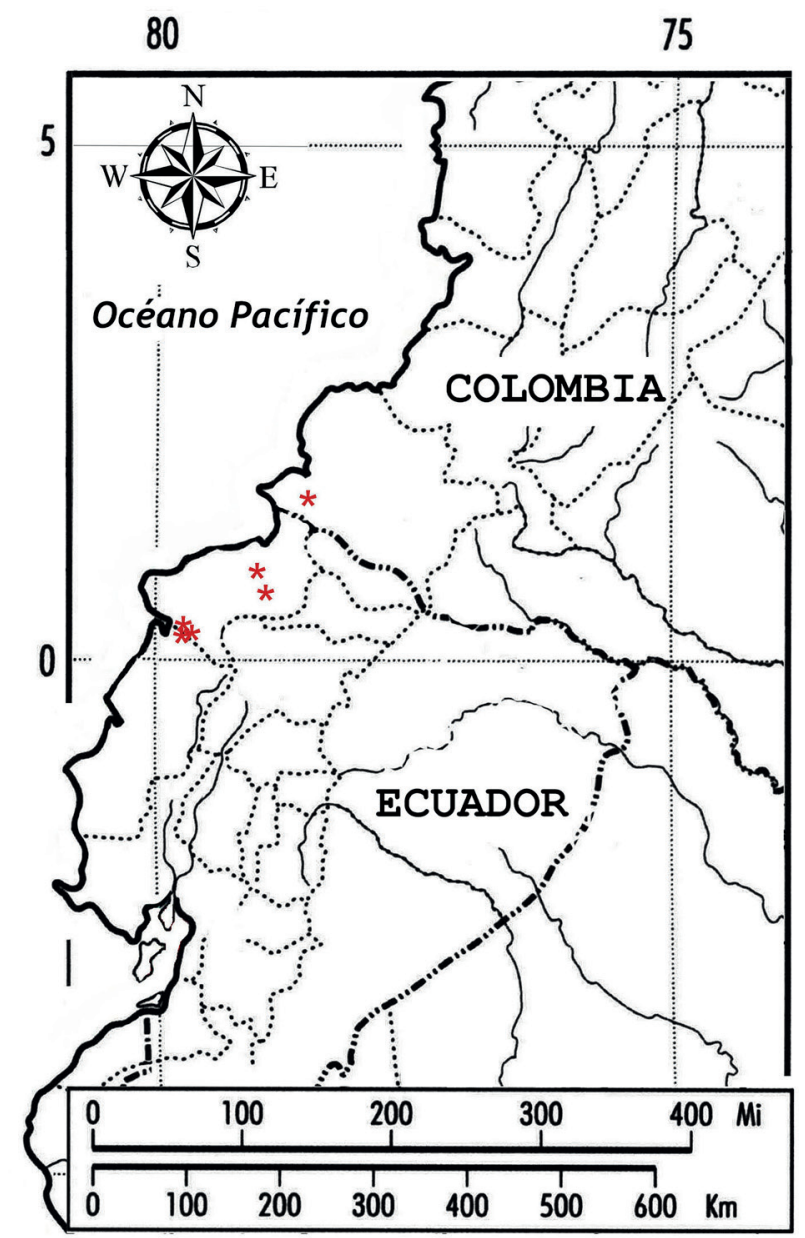

Figura 5: Mapa de distribución de Quararibea calycoptera Fern.Alonso \& Cornejo (asteriscos color rojo).

se separa bien por los caracteres que se señalan en el Cuadro 1. La diferente consistencia de la lámina foliar (membranácea), los pedicelos florales claramente más largos con las bractéolas más largas, erecto-patentes y dispersas a lo largo del pedicelo, sus cálices de mayor tamaño anchamente tubular-campanulados y con cinco alas llamativamente elevadas de 2-3 mm, permiten separar sin dificultad a $Q$. calycoptera de Q. grandifolia (Figs. 2C, 3C). La otra especie asignable también al grupo de $Q$. grandifolia (Cuadro 1), Q. cornejoi, se separa fácilmente por los caracteres foliares (hojas también membranáceas, pero estrechamente oblanceoladas $9-22 \times 4-5 \mathrm{~cm}$ y gruesamente bulladas), el pedicelo floral más corto (3-4 $\mathrm{mm}$ ) y con bractéolas mucho menores (1-2 mm), agrupadas en posición media o distal, y el cáliz floral, tubular-cilíndrico, menor $(8-10 \times 3-3.5 \mathrm{~mm})$ con alas muy poco resaltadas $(<1 \mathrm{~mm})$. Aunque el tipo de Q. grandifolia fue descrito con un cáliz provisto de diez alas (Little, 1948), del estudio de este material y de las nuevas recolecciones de la especie, se concluye que el número usual de alas completas es de cinco, frecuentemente acompañadas de (0-)2-4 alas incipientemente desarrolladas, intercaladas con las anteriores. Esto ocurre también en la especie que nos ocupa ( $Q$. calycoptera) -como se muestra en el corte transversal del cáliz que aparece en la Figura 3A-, y en las imágenes que aparecen en el protólogo de la especie recientemente descrita $Q$. cornejoi (Fernández-Alonso, 2021b).

\section{Contribución de autores}

JLF concibió el trabajo y escribió la primera versión del manuscrito. XC contribuyó con JLF en la obtención de datos de campo, la revisión de herbarios, la diagnosis de la nueva especie y en la revisión del manuscrito.

\section{Financiamiento}

Este estudio se llevó a cabo gracias al apoyo del Real Jardín Botánico del Consejo Superior de Investigaciones Científicas (CSIC) y al Ministerio de Ciencia e Innovación de España por facilitar los trabajos de revisión en plantas tropicales con cargo a los proyectos: Intramural CSIC: 2009301071 y CGL2010-19747 (subprograma BOS), ambos anteriores a 2016. También el Instituto de Ciencias Naturales de la Universidad Nacional de Colombia y su herbario COL apoyaron los trabajos de campo y de revisión relacionados con la familia Malvaceae s.l.

\section{Agradecimientos}

Para el presente trabajo fueron fundamentales las muestras recibidas para identificación, así como los préstamos remitidos en los años pasados, por colegas de los siguientes herbarios: CUVC (P. Silverstone ( $\mathrm{T}$ ), GUAY (Carmen Bonifaz), MO (J. Solomon), PSO (B. Ramirez) y QCNE (D. Neill). Muchas de las colecciones recibidas como obsequio para identificación quedaron depositadas en los herbarios COL y MA. Al margen de las fuentes e instituciones que apoyaron esta investigación, JLF deja constancia de su agradecimiento a los curadores temporales de los herbarios COL, Carlos 
Cuadro 1: Características morfológicas de Quararibea calycoptera Fern.Alonso \& Cornejo, comparada con otras especies relacionadas.

\begin{tabular}{|c|c|c|c|}
\hline \multicolumn{4}{|c|}{ Especies } \\
\hline Caracteres & Q. calycoptera Fern.Alonso \& Cornejo & Q. cornejoi Fern.Alonso & Q. grandifolia (Little) Cuatrec. \\
\hline Lámina consistencia & membranácea & membranácea & cartácea \\
\hline Pedicelo floral & $8-18 \mathrm{~mm}$ & $3-4 \mathrm{~mm}$ & $4-7 \mathrm{~mm}$ \\
\hline Bractéolas ubicación & en pedicelo medio o distal & dispersas en pedicelo & agrupadas en posición distal \\
\hline Bractéolas (longitud) & 5-11 mm & $1-2 \mathrm{~mm}$ & 3-6 mm \\
\hline Cáliz forma & anchamente tubular-campanulado & tubular-cilíndrico & estrechamente tubular-campanulado \\
\hline Cáliz dimensiones & $27-31 \times 10-12 \mathrm{~mm}$ & $8-10 \times 3-3.5 \mathrm{~mm}$ & $20-25 \times 7-9 \mathrm{~mm}$ \\
\hline Cáliz alas (alto) & $2-3 \mathrm{~mm}$ & $0.3-1 \mathrm{~mm}$ & $1-1.5 \mathrm{~mm}$ \\
\hline
\end{tabular}

Parra, y MA, Mauricio Velayos, por facilitar la recepción y envío de préstamos de las colecciones en estudio de los géneros Matisia y Quararibea. El segundo autor agradece a la familia Cabrera, por haber proporcionado hospedaje a los colectores del tipo en el área de Mache-Chindul. Nuestro agradecimiento a los revisores y editores de este manuscrito por los valiosos comentarios y observaciones efectuadas, que ayudaron a mejorarlo.

\section{Literatura citada}

Alverson, W. S. 1989. Matisia and Quararibea (Bombacaceae) should be retained as separate genera. Taxon 38(3): 377388. DOI: https://doi.org/10.2307/1222268

Cascante-Marín, A., J. Sánchez-González y W. S. Alverson. 2018. A New Quararibea (Malvaceae) from the Caribbean Lowlands of Costa Rica. Novon 26(3): 262-267. DOI: https://doi. org/10.3417/2018106

Cornejo, X. 2011. Endemismo en la región Litoral. In: León-Yánez, S., R. Valencia, N. Pitman, L. Endara, C. Ulloa-Ulloa y H. Navarrete (eds.). Libro rojo de las plantas endémicas del Ecuador, 2a ed. Publicaciones del Herbario QCA, Pontificia Universidad Católica del Ecuador. Quito, Ecuador. Pp. 25-28.

Dodson, C. H. y A. H. Gentry. 1991. Biological extinction in western Ecuador. Annals of the Missouri Botanical Garden 78(2): 273-295. DOI: https://doi.org/10.2307/2399563

Fernández-Alonso, J. L. 1996. Contribuciones al conocimiento del género Phragmotheca Cuatrec. (BombacaceaeQuararibeae). Caldasia 18(3): 253-284.

Fernández-Alonso, J. L. 1999. Nueva especie y notas del género Quararibea (Bombacaceae). Revista de la Academia
Colombiana de Ciencias Exactas, Físicas y Naturales 23 (Suplemento especial): 49-52.

Fernández-Alonso, J. L. 2001a. Bombacaceae neotropicae novae vel minus cognitae $I$. Novedades taxonómicas y corológicas en Matisia, Quararibea y Spirotheca. Revista de la Academia Colombiana de Ciencias Exactas, Físicas y Naturales 25(95): 183-206.

Fernández-Alonso, J. L. 2001b. Bombacaceae neotropicae novae vel minus cognitae II. Novedades taxonómicas y corológicas en Matisia y Quararibea del norte de Sudamérica. Caldasia 23(2): 351-382.

Fernández-Alonso, J. L. 2016. Quararibea. (Malvaceae). In: Bernal, R., S. R. Gradstein y M. Celis (eds.). Catálogo de plantas y líquenes de Colombia, Vol. 2. Instituto de Ciencias Naturales, Universidad Nacional de Colombia. Bogotá, Colombia. Pp. 1535-1570.

Fernández-Alonso, J L. 2021a. Matisia, Phragmotheca y Quararibea. In: Bernal, R., S. R. Gradstein y M. Celis (eds.). Catálogo de plantas y líquenes de Colombia. Instituto de Ciencias Naturales, Universidad Nacional de Colombia. Bogotá, Colombia. http:// catalogoplantasdecolombia.unal.edu.co (consultado enero de 2021).

Fernández-Alonso J. L. 2021b. Malvaceae Neotropicae novae vel minus cognitae X. Nuevas especies de Quararibea de Colombia y Ecuador. Anales del Jardín Botánico de Madrid 78(2): e112.

Fernández Alonso, J. L. y S. Castroviejo. 2001. Bombacaceae neotropicae novae vel minus cognitae IV. De Matisiis et Quararibeis nonnullis in provincia chocoana regni novogranatensis provenientibus. Anales del Jardín Botánico de Madrid 59(1): 152-156. 
Font Quer, P. 1985. Diccionario de Botánica. Editorial Labor. Barcelona, España. 1244 pp.

Harris, J. G. y M. W. Harris. 2001. Plant identification terminology: An illustrated glossary. 2nd ed. Spring Lake Publishing. Spring Lake, USA. 206 pp.

Ibáñez, A., S. Castroviejo, J. L. Fernández-Alonso y M. Correa. 2005. Catálogo de la flora de la región de Bahía Honda (Veraguas, Panamá). In: Castroviejo, S. y A. Ibáñez (eds.). Estudios sobre la biodiversidad de la región de Bahía Honda (Veraguas, Panamá). Biblioteca de Ciencias 20. Consejo Superior de Investigaciones Científicas. Madrid, España. Pp. 177-317.

Jørgensen, P. y S. León (eds.). 1999. Catalogue of the Vascular Plants of Ecuador. Monographs in Systematic Botany from the Missouri Botanical Garden 75. Missouri Botanical Garden Press. Saint Luis, Missouri, USA.

IUCN. 2019. Guidelines for using the International Union for Conservation of Nature, Red List Categories and Criteria, Version 14, prepared by the Standards and Petitions
Committee. Gland, Suiza. http://www.iucnredlist.org/ documents/RedListGuidelines.pdf (consultado junio de 2021).

JSTOR. 2021. Global Plants database JSTOR. https://plants.jstor. org/ (consultado marzo de 2021).

Little, Jr., E. L. 1948. New species of trees from western Ecuador. Journal of the Washington Academy of Sciences 38(3): 87105.

Little, E. L. y R. G. Dixon. 1969. Árboles comunes de la provincia de Esmeraldas, Estudio de Preinversión para el desarrollo forestal del Noroccidente de Ecuador, Informe final, Tomo IV. Food and Agriculture Organization. Roma, Italia. 1-532.

Thiers, B. 2020. Index Herbariorum: A global directory of public herbaria and associated staff. New York Botanical Garden's Virtual Herbarium. New York, USA. http://sweetgum.nybg. org/science/ih/ (consultado marzo de 2021).

TROPICOS. 2021. Tropicos.org. Missouri Botanical Garden. Missouri, USA. http://www.tropicos.org/Name/ (consultado marzo de 2021). 